\title{
Nonsurgical Management Following Local Resection for Early Rectal Cancer in Patients with High-risk Factors: A Single-institute Experience
}

\author{
Daisuke Nishizaki ${ }^{1)}$, Nobuaki Hoshino ${ }^{1 / 2)}$, Koya Hida ${ }^{1)}$, Yoshitaka Nishikawa ${ }^{2 / 3)}$, Takahiro Horimatsu ${ }^{3}$, \\ Sachiko Minamiguchi ${ }^{4)}$, Katsuyuki Sakanaka ${ }^{5)}$ and Yoshiharu Sakai ${ }^{1)}$ \\ 1) Department of Surgery, Kyoto University Graduate School of Medicine, Kyoto, Japan \\ 2) Department of Health Informatics, School of Public Health, Kyoto University, Kyoto, Japan \\ 3) Department of Therapeutic Oncology, Graduate School of Medicine, Kyoto University, Kyoto, Japan \\ 4) Department of Diagnostic Pathology, Kyoto University Hospital, Kyoto, Japan \\ 5) Department of Radiation Oncology and Image-Applied Therapy, Kyoto University Hospital, Kyoto, Japan
}

\begin{abstract}
Objective: Additional surgery is considered for patients at high risk for lymph node metastasis (LNM) after local resection for early rectal cancer. Several factors are considered as indications for additional surgery, although there are currently no definitive criteria. This study aimed to clarify the need for additional surgery based on the number of risk factors for LNM and to evaluate the significance of submucosal invasion on recurrence.

Methods: Patients with early rectal cancer harboring risk factors for LNM who underwent local resection between March 2005 and December 2016 were retrospectively analyzed. Associations among the number of risk factors, prognosis, and additional treatment after local resection were investigated.

Results: A total of 29 eligible patients were classified into the surgery $(\mathrm{n}=10)$, chemoradiotherapy $(\mathrm{n}=$ 7 ), and no-additional-treatment (NAT, $n=12$ ) groups. Among the 29 patients, 15 patients (52\%) with only one risk factor did not relapse. The NAT group harbored fewer risk factors for LNM, and 8 of the 12 patients (67\%) had only deep submucosal invasion. Local recurrence occurred in one patient in the chemoradiotherapy group. The estimated 5-year overall survival rates were $88.9 \%, 75.0 \%$, and $81.5 \%$ in the surgery, chemoradiotherapy, and NAT groups, respectively. There were no disease-specific deaths in the overall cohort.

Conclusions: In the present study, no recurrence occurred in patients who did not receive additional surgery with deep submucosal invasion as the only risk factor. A multicenter investigation is necessary to confirm the safety of nonsurgical options.
\end{abstract}

\section{Keywords}

rectal neoplasm, lymphatic metastasis, chemoradiotherapy, organ preservation, endoscopic mucosal resection

J Anus Rectum Colon 2020; 4(4): 174-180

\section{Introduction}

The number of local resections performed for early rectal cancer has increased in recent years, and most patients with T1 colorectal cancer can be cured by local resection[1,2]. However, lymph node metastasis (LNM) occurs in approxi- 
mately $10 \%$ of patients with $\mathrm{T} 1$ colorectal cancer[3-6]. Therefore, additional surgery is considered in patients at high risk for LNM after local resection[3,7]. According to the guidelines of the Japanese Society for the Cancer of the Colon and the Rectum (JSCCR), European Society for Medical Oncology, and the National Comprehensive Cancer Network, several factors that should be considered as risk factors for LNM or cancer recurrence include positive vertical margins, lymphatic or venous invasion, high-grade tumor budding, deep tumor invasion $(\geq 1,000 \mu \mathrm{m})$ of the submucosa, and poorly differentiated, mucinous, or signet-ring cell tumors[7-9]. These factors have been discussed during patient-clinician communications, alongside age and comorbidities[10]. However, additional surgery in patients at high risk for recurrence is controversial because the current criteria for additional surgery are not definitive. Furthermore, additional surgery for rectal cancer, such as low anterior resection and abdominoperineal resection, may be too invasive for patients with early rectal cancer as these approaches are associated with high rates of short- and long-term morbidities including anastomotic leakage, bleeding, and sexual or urinary dysfunction[11-13]. Therefore, some patients choose observation rather than additional surgery, and adjuvant chemoradiotherapy has been recently discussed for high-risk patients who wish to avoid additional surgery[14,15]. However, the outcomes of patients who do not undergo additional surgery remain unclear. This study aimed to assess the outcomes of these patients and to investigate the need for additional surgery based on the number of risk factors in patients with early rectal cancer.

\section{Methods}

\section{Patients}

This descriptive, exploratory analysis of a single-center series included patients who underwent local resection for pathological stage T1 rectal cancer between March 2005 and December 2016. Patients with rectal cancer located in RS, $\mathrm{Ra}$, and $\mathrm{Rb}$ were included in the study[16]. The exclusion criteria were 1) advanced cancer invading beyond the muscularis propria, 2) cancer without any high-risk factors, and 3) clinical LNM on computed tomography (CT) images assessed by a multidisciplinary team before additional treatment. In the present study, endoscopic submucosal dissection (ESD), endoscopic mucosal resection (EMR), and transanal minimally invasive surgery (TAMIS) were defined as procedures for local resection[17,18]. The choice of local resection procedure was based on several factors including tumor size, tumor location, patient age, and comorbidities. The study was approved by the Ethics Committee of Kyoto University (certified number: R1447). Individual consent was not required because of the retrospective nature of the study.

\section{Treatment and follow-up}

Additional surgery was recommended for patients with risk factors according to the JSCCR guidelines. In patients who wished to avoid surgery, the decision to undergo treatment with chemoradiotherapy or to undergo observation was based on the number of risk factors, the type of risk factors, and patient preferences. All patients were followed every 3 months for the first 3 years after completion of additional treatment. Physical and digital examination, colonoscopy, chest and abdominal CT, and blood test for carcinoembryonic antigen were performed according to the surveillance schedule of the JSCCR guidelines[7]. Magnetic resonance imaging was not routinely performed. Patients without signs of recurrence after the first 3 years were followed every 6 months thereafter.

\section{Risk stratification}

The patient characteristics and details of additional treatments were retrospectively reviewed using data obtained from the electronic medical records. The patient characteristics included age, sex, tumor size, grade of tumor budding, tumor differentiation, lymphatic and venous invasion, vertical and horizontal margins of the resected specimen, and tumor depth. Presence of the following risk factors was determined in all patients: positive vertical margins, lymphatic or venous invasion, high-grade tumor budding, deep tumor invasion $(\geq 1,000 \mu \mathrm{m})$ of the submucosa, and poorly differentiated, mucinous, or signet-ring cell tumors. The grade of tumor budding was missing in three patients.

\section{Statistical analysis}

Categorical variables were described as frequencies and percentages and analyzed using Fisher's exact test. Continuous variables were described as medians with ranges and analyzed using the Kruskal-Wallis test. Overall survival (OS) and relapse-free survival (RFS) were estimated using the Kaplan-Meier method and analyzed using the log-rank test. OS was defined as the time from the date of initial local resection to the date of death. RFS was defined as the time from the date of local resection to the date of recurrence or death due to any cause. Imputation for tumor budding to create a worst-case scenario was performed as a sensitivity analysis. All statistical analyses were performed with $\mathrm{R}$ version 3.4.4[19]. All $P$ values were two-sided, and a $P$ value < 0.05 was considered to be statistically significant.

\section{Results}

\section{Patient characteristics}

A total of 35 patients who underwent local resection for 


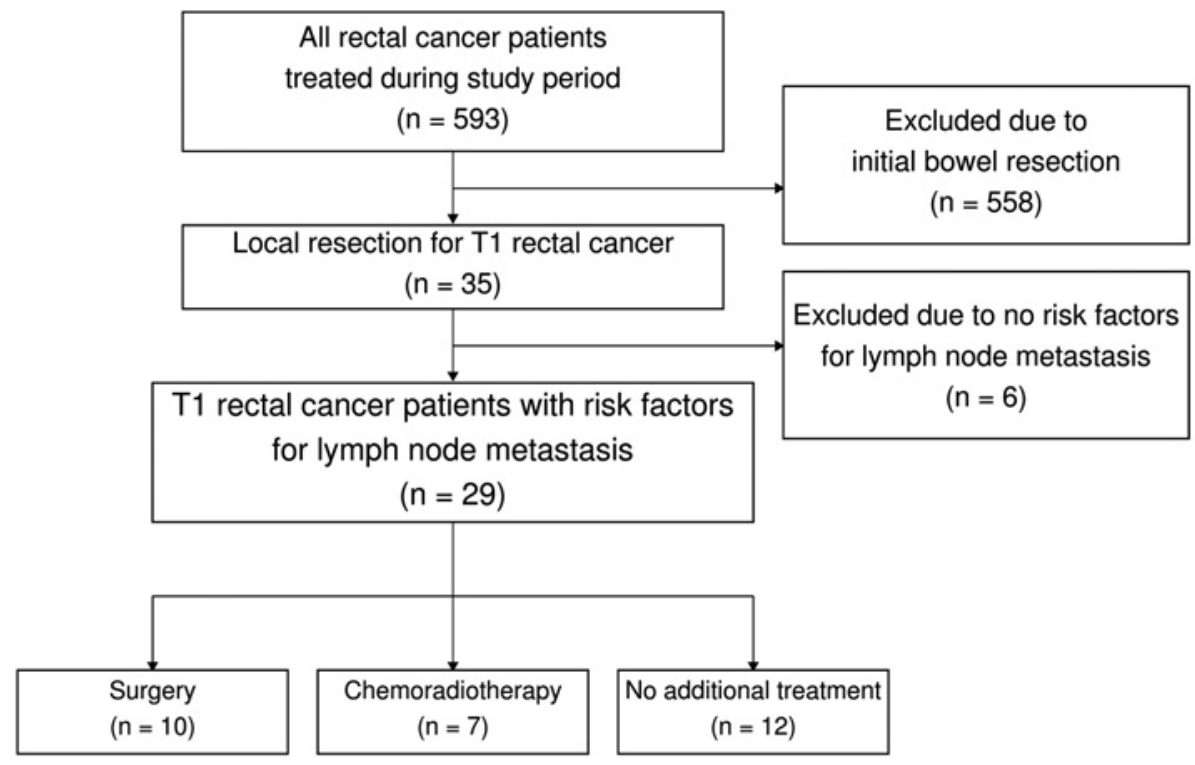

Figure 1. Flow diagram for patient selection.

T1 rectal cancer were identified among 593 patients with rectal cancer treated during the study period. After the exclusion of 6 patients with no risk factors for recurrence, a total of 29 patients harboring risk factors for LNM were included in the study (Figure 1). The patient characteristics are listed in Table 1. The local resection approaches were ESD, EMR, and TAMIS in 17 (59\%), $9(31 \%)$, and $3(10 \%)$ patients, respectively. The median follow-up period was 35 (range: 12-111) months. In the overall cohort of 29 patients, $15(52 \%), 7(24 \%), 4(14 \%)$, and $3(10 \%)$ patients harbored $1,2,3$, and 4 risk factors, respectively. Of note, 12 of the 15 patients with one risk factor had deep submucosal invasion (Figure 2).

\section{Details of additional treatments}

In the present study cohort, 10 patients (35\%) underwent surgery, and 7 patients (24\%) underwent chemoradiotherapy as additional treatment. The remaining 12 patients (41\%) did not receive additional treatment (no-additional-treatment [NAT] group) (Table 2). In the surgery group, six patients underwent laparoscopic low anterior resection, whereas four patients underwent laparoscopic intersphincteric resection. In all patients, postoperative pathological findings indicated no residual tumor cells in the resected specimens. Minor anastomotic leakage occurred in two patients who underwent intersphincteric resection. Details of patients in the chemoradiotherapy group are presented in Table 3. In patients with clear resection margins, 45-50.4 Gy was delivered in 25-28 fractions to the mesorectum and pelvic side walls where microscopic involvement was a concern. In patients with positive vertical margins, boost radiotherapy up to a total dose of 58-59.8 Gy was administered. Concurrent chemotherapy was 5-FU until 2011 and capecitabine from 2012, except for one patient who was administered irinotecan because a regimen for advanced cancer was considered appropriate for a positive vertical margin.

\section{Details of risk factors}

The number of risk factors was larger in the surgery and chemoradiotherapy groups than in the NAT group $(P=$ 0.007) (Table 2). Eleven of the 12 patients in the NAT group harbored one risk factor, including eight patients with deep tumor invasion and three patients with lymphatic or venous invasion (Figure 2). High-grade tumor budding and poorly differentiated tumors were not observed in the NAT group. In sensitivity analysis with the worst-case scenario, the number of risk factors remained larger in the surgery and chemoradiotherapy groups than in the NAT group $(P=$ 0.012).

\section{Survival analysis based on additional treatments}

In the chemoradiotherapy group, local intraluminal recurrence, following the completion of chemoradiotherapy, occurred 11 months after local resection in one patient with multiple risk factors including positive vertical margins. The patient did not undergo salvage surgery because of comorbidities and died from hepatocellular carcinoma 31 months following the local resection. There were no distant metastases in any of the study patients. During the study period, five patients (17\%) died from causes other than rectal cancer recurrence, including myocardial infarction, lung cancer, hepatocellular carcinoma, and ureteral cancer. The 5-year OS rates were $88.9 \%$ (95\% confidence interval [CI]: 70.6-100), 75.0\% (95\% CI: 42.6-100), and 81.5\% (95\% CI: 61.1-100) in the surgery, chemoradiotherapy, and NAT groups, respectively $(P=0.825)$ (Figure 3$)$. Conversely, the 5-year RFS 
Table 1. Patient Characteristics.

\begin{tabular}{|c|c|}
\hline No. of patients & 29 \\
\hline Age (years), median (range) & $68(35-82)$ \\
\hline Follow-up time (months), median (range) & $35(12-111)$ \\
\hline Tumor size $(\mathrm{mm})$, median (range) & $18(5-52)$ \\
\hline Females, n $(\%)$ & $9(31)$ \\
\hline \multicolumn{2}{|l|}{ Local resection procedure, $\mathrm{n}(\%)$} \\
\hline ESD & $17(59)$ \\
\hline EMR & $9(31)$ \\
\hline TAMIS & $3(10)$ \\
\hline \multicolumn{2}{|l|}{ Tumor configuration, $\mathrm{n}(\%)$} \\
\hline Pedunculated & $13(46)$ \\
\hline Non-pedunculated & $15(54)$ \\
\hline \multicolumn{2}{|l|}{ Tumor location, n (\%) } \\
\hline RS & $4(14)$ \\
\hline $\mathrm{Ra}$ & $6(21)$ \\
\hline $\mathrm{Rb}$ & $19(66)$ \\
\hline \multicolumn{2}{|l|}{ Pathology findings, n (\%) } \\
\hline Submucosal invasion $\geq 1,000 \mu \mathrm{m}$ & $25(86)$ \\
\hline Positive vertical margin & $7(24)$ \\
\hline Lymphatic invasion & $6(21)$ \\
\hline Venous invasion & $9(31)$ \\
\hline Budding grade $2 / 3$ & $3(10)$ \\
\hline Poorly differentiated, mucinous, or signet-ring cell tumors & $3(10)$ \\
\hline \multicolumn{2}{|l|}{ No. of risk factors, $\mathrm{n}(\%)$} \\
\hline 1 & $15(52)$ \\
\hline 2 & $7(24)$ \\
\hline 3 & $4(14)$ \\
\hline 4 & $3(10)$ \\
\hline
\end{tabular}

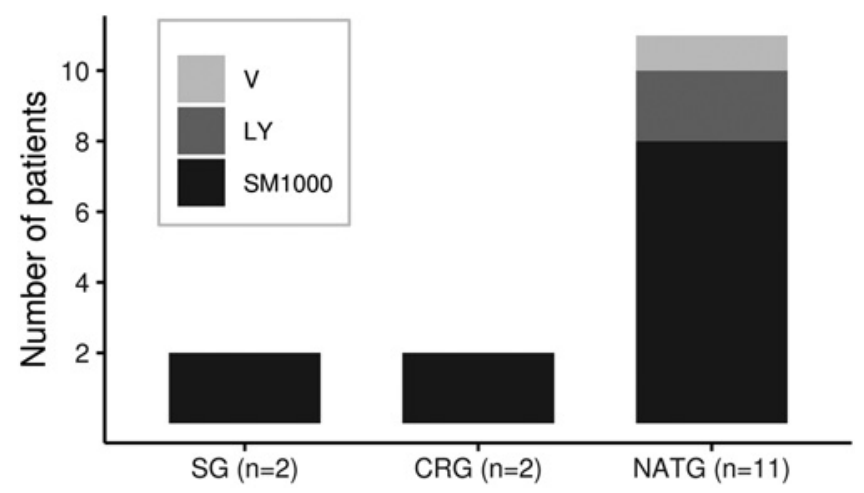

Figure 2. Details of risk factors in patients with one risk factor. LY, lymphatic invasion; V, venous invasion; SM1000, submucosal invasion $\geq 1,000 \mu \mathrm{m}$; SG, surgery group; CRG, chemoradiotherapy group; NATG, no additional treatment group

rates were $88.9 \%$ (95\% CI: 70.6-100), 85.7\% (95\%CI: 63.3 $100)$, and $81.5 \%$ (95\%CI: 61.1-100) in the surgery, chemoradiotherapy, and NAT groups, respectively $(P=$ 0.867) (Figure 3). There were no disease-specific deaths in the overall study cohort.

\section{Discussion}

In the present study, we investigated the association between the number of risk factors for LNM and additional treatment approaches after local resection. Patients with one risk factor for LNM tended to receive no additional treatment, whereas those with multiple risk factors were more likely to undergo surgery or chemoradiotherapy. There were no disease-specific deaths in any of the groups and no significant differences in OS and RFS among the three groups.

Several guidelines, which suggest the aforementioned risk factors for LNM, also indicate that additional surgery may not always be necessary in patients with deep submucosal invasion $\geq 1,000 \mu \mathrm{m}[7-9]$. In the present study, 8 of the 12 patients in the NAT group who harbored deep submucosal invasion $\geq 1,000 \mu \mathrm{m}$ as the only risk factor were free from recurrence without additional treatment. Our analysis based on the number of risk factors revealed that 15 patients with one risk factor, including 12 patients with deep submucosal 
Table 2. Clinical Characteristics.

\begin{tabular}{|c|c|c|c|c|}
\hline & $\begin{array}{l}\text { Surgery } \\
\text { group }\end{array}$ & $\begin{array}{l}\text { Chemoradiotherapy } \\
\text { group }\end{array}$ & $\begin{array}{l}\text { No-additional-treatment } \\
\text { group }\end{array}$ & $P$ value \\
\hline Number of patients & 10 & 7 & 12 & \\
\hline Age (years), median (range) & $65(48-81)$ & $68(35-82)$ & $70.5(57-82)$ & 0.362 \\
\hline Follow-up time (months), median (range) & $32.5(21-89)$ & $31(12-63)$ & $48.5(14-111)$ & 0.433 \\
\hline Tumor size (mm), median (range) & $21(5-40)$ & $18.5(8-35)$ & $15(10-52)$ & 0.964 \\
\hline Female, $\mathrm{n}(\%)$ & $5(50)$ & $1(14)$ & $4(33)$ & 0.382 \\
\hline Recurrence, n (\%) & $0(0)$ & $1(14)$ & $0(0)$ & 0.241 \\
\hline \multicolumn{5}{|l|}{ Pathology findings, $\mathrm{n}(\%)$} \\
\hline Submucosal invasion $\geq 1,000 \mu \mathrm{m}$ & $9(90)$ & $7(100)$ & $9(75)$ & 0.415 \\
\hline Positive vertical margin & $4(40)$ & $2(29)$ & $1(8)$ & 0.224 \\
\hline Lymphatic invasion & $2(20)$ & $2(29)$ & $2(17)$ & 0.854 \\
\hline Venous invasion & $5(50)$ & $3(43)$ & $1(8)$ & 0.077 \\
\hline Budding grade $2 / 3$ & $2(20)$ & $1(14)$ & $0(0)$ & 0.315 \\
\hline Poorly differentiated, mucinous, or signet-ring cell tumors & $2(20)$ & $1(14)$ & $0(0)$ & 0.315 \\
\hline Number of risk factors, $\mathrm{n}(\%)$ & & & & 0.007 \\
\hline 1 & $2(20)$ & $2(29)$ & $11(92)$ & \\
\hline 2 & $4(40)$ & $2(29)$ & $1(8)$ & \\
\hline 3 & $2(20)$ & $2(29)$ & $0(0)$ & \\
\hline 4 & $2(20)$ & $1(14)$ & $0(0)$ & \\
\hline
\end{tabular}

Table 3. Characteristics of Patients Who Underwent Chemoradiotherapy.

\begin{tabular}{|c|c|c|c|c|c|c|c|c|c|c|c|c|c|}
\hline Sex & Age & Histology & $\begin{array}{c}\text { Radiation } \\
\text { dose } \\
(\mathrm{Gy})\end{array}$ & $\begin{array}{l}\text { Concomitant } \\
\text { chemotherapy } \dagger\end{array}$ & $\begin{array}{l}\text { Fraction } \\
\text { (times) }\end{array}$ & $\begin{array}{l}\text { Diameter } \\
(\mathrm{mm})\end{array}$ & $\begin{array}{c}\text { Depth of } \\
\text { submucosal } \\
\text { invasion }\end{array}$ & VM & $\begin{array}{c}\text { Budding } \\
\text { grade } \\
2 / 3\end{array}$ & LY & V & $\begin{array}{c}\text { Number } \\
\text { of risks }\end{array}$ & Recurrence \\
\hline Male & 82 & Tub & 59.4 & $\begin{array}{c}\text { 5-FU } \\
800 \mathrm{mg} / \text { body }\end{array}$ & 33 & 15 & $\geq 1,000 \mu \mathrm{m}$ & + & - & + & + & 4 & Local \\
\hline Male & 74 & Tub & 45 & Cape $1650 \mathrm{mg} / \mathrm{m}^{2}$ & 25 & 16 & $7,000 \mu \mathrm{m}$ & - & - & - & - & 1 & - \\
\hline Female & 54 & Tub & 50.4 & Cape $1650 \mathrm{mg} / \mathrm{m}^{2}$ & 28 & 35 & $\geq 1,000 \mu \mathrm{m}$ & - & - & - & - & 1 & - \\
\hline Male & 68 & Tub & 45 & Cape $1650 \mathrm{mg} / \mathrm{m}^{2}$ & 25 & 21 & $4,000 \mu \mathrm{m}$ & - & + & + & - & 3 & - \\
\hline Male & 67 & Tub & 45 & Cape $1650 \mathrm{mg} / \mathrm{m}^{2}$ & 25 & 8 & $5,200 \mu \mathrm{m}$ & - & - & - & + & 2 & - \\
\hline Male & 74 & Tub & 58 & $\begin{array}{l}\text { CPT-11 } 60 \mathrm{mg} / \mathrm{m}^{2} \\
\mathrm{~S}-1120 \mathrm{mg} / \mathrm{body}\end{array}$ & 29 & unknown & $\geq 1,000 \mu \mathrm{m}$ & + & - & - & + & 3 & - \\
\hline Male & 35 & Por & 45 & Cape 1650 mg/m² & 25 & 30 & $\geq 1,000 \mu \mathrm{m}$ & - & - & - & - & 2 & - \\
\hline
\end{tabular}

Abbreviations: VM, vertical margin; LY, lymphatic invasion; V, venous invasion; Cape, capecitabine; 5-FU, 5-fluorouracil; Tub, tubular adenocarcinoma; Por, poorly differentiated adenocarcinoma

$\dagger$ Daily chemotherapy dose
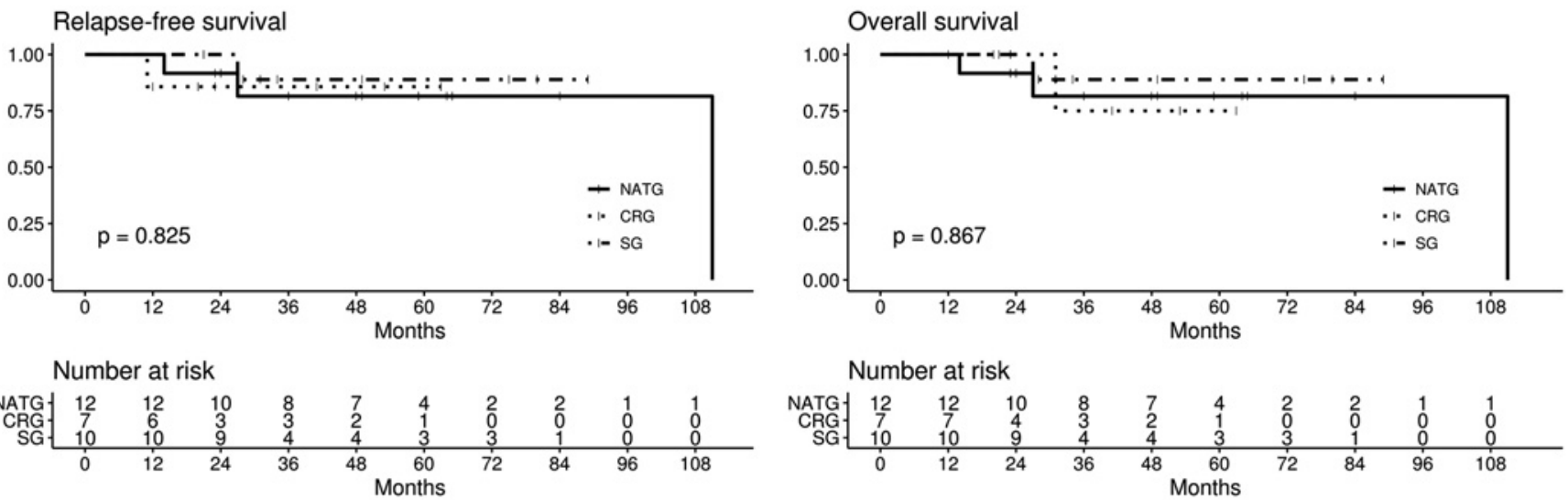

Figure 3. Kaplan-Meier survival curves.

SG, surgery group; CRG, chemoradiotherapy group; NATG, no additional treatment group 
invasion alone, did not relapse regardless of additional treatment. These results suggest that the impact of deep submucosal invasion on LNM might be different from the impact of other risk factors. Although lymphatic or venous invasion, high-grade tumor budding, and poorly differentiated, mucinous, or signet-ring cell tumors are direct risk factors for LNM, deep submucosal invasion might be a sentinel and not a direct risk factor for LNM. The risk is higher for a tumor extending into the submucosal layer of the rectum to encounter and invade the numerous vessels residing in the submucosal tissue[20]. However, lymphatic vessels are less dense in the deepest two-thirds than in the superficial onethird of the submucosa[20]. In addition, the number and size of blood vessels do not increase with the depth of submucosa[21]. Therefore, deep submucosal invasion without lymphatic or venous invasion cannot explain the increased risk of LNM, and deep submucosal invasion alone may not be a direct risk factor for LNM. Furthermore, a recent study reported that the proportion of pathological LNM was only $1.3 \%$ (95\% CI: $0.6 \%-2.5 \%$ ) in patients with pathological T1 colorectal cancer harboring only deep submucosal invasion[22,23]. Given that there were no disease-specific deaths in the present study cohort, patients with deep submucosal invasion alone might not require additional treatment after local resection, although careful observation is mandatory.

In the present study, chemoradiotherapy was administered in patients who wished to avoid surgery despite multiple risk factors. Among seven patients who underwent chemoradiotherapy, one patient who had four risk factors, including positive vertical margins, developed local recurrence, which was limited to the rectum and could potentially be surgically salvaged. However, the patient was clinically vulnerable with multiple comorbidities, and salvage surgery was not performed. Conversely, the other patient with positive vertical margins did not relapse during the 2-year follow-up period. The efficacy of chemoradiotherapy may depend on the responsiveness of the tumor. The outcomes of patients with positive vertical margins who avoid surgery can be evaluated only in retrospective studies because of the rarity of the clinical condition and the ethical issues related to prospective studies, highlighting the value of retrospective series.

Consideration of risk factors other than positive vertical margins suggests that the risk of local recurrence is higher in patients with pathological $\mathrm{T} 1$ rectal cancer than in those with pathological $\mathrm{T} 1$ colon cancer in the absence of additional treatment[24]. In a large series of patients with pT1 rectal tumors, pathological LN metastases were more common in the rectal cancer than in the colon cancer[25]. However, chemoradiotherapy might aid in preventing local recurrence in rectal cancer[14]. To achieve organ preservation, chemoradiotherapy might be a suitable substitute for radical surgery in patients with few risk factors, although the current series with a small number of cases cannot provide con- clusive evidence.

The present study has several limitations. First, the study was retrospectively conducted at a single center and included a small series of patients, and selection bias cannot be denied. Second, the median observational period of 35 months was relatively short and that of the chemoradiotherapy group was shorter than those of the other groups, and the long-term outcomes were not evaluated. Third, pelvic magnetic resonance imaging was not routinely used for the assessment of staging and recurrence. We acknowledge that the study sample size was too small to reach conclusive results. However, identification of low-risk patients is an urgent task because of the paucity of evidence on alternative treatment approaches in patients with early rectal cancer. It is, therefore, important for one to conduct additional retrospective series before initiating large-scale studies.

In conclusion, no recurrence occurred in patients who did not receive additional surgery with deep submucosal invasion as the only risk factor. Since the number of patients diagnosed with early rectal cancer is small, a multicenter collaborative study is important for confirmation of the current study's conclusions.

\section{Conflicts of Interest}

There are no conflicts of interest.

\section{Source of Funding}

This study was supported by Grants-in-Aid for Scientific Research from the JSCO Clinical Research Grant Program 2017.

\section{Author Contributions}

YS conceived the study. DN, KS, and $\mathrm{KH}$ designed the study protocol. YN, TH, DN, and YS collected the patient data. SM performed the histological examination of the rectal specimen. DN analyzed and interpreted the patient data and was a major contributor in writing the manuscript. DN, $\mathrm{NH}$, and $\mathrm{KH}$ coordinated the manuscript. $\mathrm{KS}, \mathrm{TH}$, and $\mathrm{YN}$ provided specific advice on the manuscript. All authors took part in the discussion of the study and approved the final manuscript.

Approval by Institutional Review Board (IRB)

This study was approved by the Ethics Committee of Kyoto University (certified number: R1447).

\section{References}

1. Yoshii S, Nojima M, Nosho K, et al. Factors associated with risk for colorectal cancer recurrence after endoscopic resection of $\mathrm{T} 1$ tumors. Clin Gastroenterol Hepatol. 2014 Feb; 12(2): 292-302.

2. You YN, Baxter NN, Stewart A, et al. Is the increasing rate of local excision for stage I rectal cancer in the United States justified?: a nationwide cohort study from the National Cancer Data- 
base. Ann Surg. 2007 May; 245(5): 726-33.

3. Kitajima K, Fujimori T, Fujii S, et al. Correlations between lymph node metastasis and depth of submucosal invasion in submucosal invasive colorectal carcinoma: a Japanese collaborative study. J Gastroenterol. 2004 Jun; 39(6): 534-43.

4. Egashira Y, Yoshida T, Hirata I, et al. Analysis of pathological risk factors for lymph node metastasis of submucosal invasive colon cancer. Mod Pathol. 2004 May; 17(5): 503-11.

5. Kobayashi H, Mochizuki $H$, Kato $T$, et al. Is total mesorectal excision always necessary for T1-T2 lower rectal cancer? Ann Surg Oncol. 2010 Apr; 17(4): 973-80.

6. Nascimbeni R, Burgart LJ, Nivatvongs $S$, et al. Risk of lymph node metastasis in $\mathrm{T} 1$ carcinoma of the colon and rectum. Dis Colon Rectum. 2002 Feb; 45(2): 200-6.

7. Watanabe T, Muro K, Ajioka Y, et al. Japanese Society for Cancer of the Colon and Rectum (JSCCR) guidelines 2016 for the treatment of colorectal cancer. Int J Clin Oncol. 2018 Feb; 23(1): 1-34.

8. Glynne-Jones R, Wyrwicz L, Tiret E, et al. Rectal cancer: ESMO Clinical Practice Guidelines for diagnosis, treatment and followup. Ann Oncol. 2017 Oct; 28(suppl 4): iv22-iv40.

9. Benson AB, 3rd, Venook AP, Al-Hawary MM. Rectal Cancer, Version 2. 2018. [Internet] 12nd Jul 2018. Available from: https://ww w.nccn.org/professionals/physician_gls/pdf/rectal.pdf.

10. Nishikawa $Y$, Horimatsu $T$, Nishizaki D, et al. Qualitative and quantitative analysis of posttreatment strategy after endoscopic resection for patients with $\mathrm{T} 1$ colorectal cancer at high risk of lymph node metastasis. J Gastrointest Canc. 2020 Mar; 51(1): 242-9.

11. van der Pas MH, Haglind E, Cuesta MA, et al. Laparoscopic versus open surgery for rectal cancer (COLOR II): short-term outcomes of a randomised, phase 3 trial. Lancet Oncol. 2013 Mar; 14 (3): $210-8$.

12. Hida K, Okamura R, Sakai Y, et al. Open versus laparoscopic surgery for advanced low rectal cancer: A large, multicenter, propensity score matched cohort study in Japan. Ann Surg. 2018 Aug; 268(2): 318-24.

13. Nesbakken A, Nygaard K, Bull-Njaa T, et al. Bladder and sexual dysfunction after mesorectal excision for rectal cancer. Br J Surg. 2000 Feb; 87(2): 206-10.

14. Sasaki T, Ito Y, Ohue M, et al. Postoperative chemoradiotherapy after local resection for high-risk $\mathrm{T} 1$ to $\mathrm{T} 2$ low rectal cancer: Results of a single-arm, multi-institutional, phase II clinical trial. Dis Colon Rectum. 2017 Sep; 60(9): 914-21.

15. Balyasnikova S, Read J, Tait D, et al. The results of local excision with or without postoperative adjuvant chemoradiotherapy for early rectal cancer among patients choosing to avoid radical surgery. Colorectal Dis. 2017 Feb; 19(2): 139-47.

16. Japanese Classification of Colorectal, Appendiceal, and Anal Carcinoma: the 3d English Edition [Secondary Publication]. J Anus Rectum Colon. 2019; 3(4): 175-95.

17. Nishiyama H, Isomoto H, Yamaguchi N, et al. Endoscopic submucosal dissection for colorectal epithelial neoplasms. Dis Colon Rectum. 2010 Feb; 53(2): 161-8.

18. Yoshitomi M, Hasegawa S, Takahashi R, et al. Transanal minimally invasive surgery for local excision of early rectal tumor. Asian J Endosc Surg. 2018 May; 11(2): 182-4.

19. R Core Team. R: A Language and Environment for Statistical Computing. R Foundation for Statistical Computing, 2018. Vienna, Austria.

20. Smith KJ, Jones PF, Burke DA, et al. Lymphatic vessel distribution in the mucosa and submucosa and potential implications for $\mathrm{T}$ 1 colorectal tumors. Dis Colon Rectum. 2011 Jan; 54(1): 35-40.

21. Brown PJ, Toh EW, Smith KJ, et al. New insights into the lymphovascular microanatomy of the colon and the risk of metastases in pT1 colorectal cancer obtained with quantitative methods and three-dimensional digital reconstruction. Histopathology. 2015 Aug; 67(2): 167-75.

22. Ajioka Y, Okura Y, Ikegami M, et al. [Stratification of the risk of lymph node metastasis of T1b (SM depth of more than 1,000 $\mu \mathrm{m}$ ) colorectal cancer]. Colorectal Disease NOW 2016. Tokyo: Nihon Medical Center; 2016. p. 63-8. Japanese.

23. Hashiguchi Y, Muro K, Saito Y, et al. Japanese Society for Cancer of the Colon and Rectum (JSCCR) guidelines 2019 for the treatment of colorectal cancer. Int J Clin Oncol. 2020 Jan; 25(1): 1-42.

24. Ikematsu H, Yoda $Y$, Matsuda $T$, et al. Long-term outcomes after resection for submucosal invasive colorectal cancers. Gastroenterology. 2013 Mar; 144(3): 551-9.

25. Debove C, Svrcek M, Dumont $S$, et al. Is the assessment of submucosal invasion still useful in the management of early rectal cancer? A study of 91 consecutive patients. Colorectal Dis. 2017 Jan; 19(1): 27-37.

Journal of the Anus, Rectum and Colon is an Open Access journal distributed under the Creative Commons Attribution-NonCommercial-NoDerivatives 4.0 International License. To view the details of this license, please visit (https://creativ ecommons.org/licenses/by-nc-nd/4.0/). 